\title{
Lower dose and lower injection speed of iodinated contrast media: a new strategy to reduce the incidence rate of immediate hypersensitivity reactions
}

\author{
Ingrid B. Böhm \\ Department of Diagnostic, Interventional, and Pediatric Radiology, Inselspital, University of Bern, Bern, Switzerland
}

Correspondence to: Ingrid B. Böhm, MD. Department of Diagnostic, Interventional, and Pediatric Radiology, Inselspital, University of Bern, Freiburgstrasse 10, CH-3010 Bern, Switzerland. Email: ingrid.boehm@insel.ch.

Provenance and Peer Review: This article was commissioned by the editorial office, Quantitative Imaging in Medicine and Surgery. The article did not undergo external peer review.

Comment on: Park HJ, Son JH, Kim TB, Kang MK, Han K, Kim EH, Kim AY, Park SH. Relationship between Lower Dose and Injection Speed of Iodinated Contrast Material for CT and Acute Hypersensitivity Reactions: An Observational Study. Radiology. 2019;293:565-72.

Submitted Feb 13, 2020. Accepted for publication Feb 28, 2020.

doi: 10.21037/qims.2020.03.09

View this article at: http://dx.doi.org/10.21037/qims.2020.03.09

Immediate hypersensitivity reactions remain a problem and concern following the application of non-ionic iodinated contrast media (ICMs). Although the incidence rate of such adverse reactions is low, the frequent and intense use of ICMs compensates the low percentages. Consequently, each radiological clinic/unit has a substantial number of patients with immediate hypersensitivity reactions. As to whether it could be possible to reduce the incidence of immediate hypersensitivity reactions, is yet one great-unsolved question.

Park and colleagues addressed this central question in their recently published retrospective study (1). They analysed the effect of lowering both the dose and the injection speed rate of non-ionic ICMs for CT in adult patients. They compared the following two study periods:

(I) The control period (CT tube voltage of $120 \mathrm{kVp}$, $2 \mathrm{~mL} \mathrm{ICM} / \mathrm{kg}$ up to a maximum of $150 \mathrm{~mL}$, and an injection speed of 3 or $4 \mathrm{~mL} / \mathrm{s}$ ) examined between August 2016 and January 2017;

(II) The interventional period (CT tube voltage of $100 \mathrm{kVp}, 1.5 \mathrm{~mL} \mathrm{ICM} / \mathrm{kg}$ up to a maximum of $130 \mathrm{~mL}$, and an injection speed of 2.5 or $3 \mathrm{~mL} / \mathrm{s}$ ) between August 2017 and January 2018.

They included a substantial number of patients $(22,291$ vs. 23,352) in their study and revealed a significant $(\mathrm{P}=0.03)$ decrease of immediate hypersensitivity reactions from $1.86 \%$ (468 of 25,119 examinations; $95 \%$ CI: $1.70-2.04 \%$ ) to $1.42 \%$ (376 of 26,491 examinations; $95 \%$ CI: $1.28-1.57 \%$ ).

The authors concluded that immediate hypersensitivity reactions to ICMs are not entirely independent of dose and injection speed of the contrast agent (1). Lowering the dose and injection speed of ICMs are easy to perform measures, and should be used regularly in radiology routine settings. The general slogan 'less is more' is also true for contrastenhanced CT scans. Low-dose ICM has several advantages, the patients' organism is less loaded with contrast material, the kidney function is altered less, and finally yet importantly, the incidence of immediate hypersensitivity reactions is lowered. Every single patient benefits from lowdose ICM procedures, especially elderly and critical ill ones.

The study by Park and colleagues is very well done and written, and shows clear results (1). I congratulate the authors for their excellent work. In addition, I like to take this opportunity to comment some points:

(I) What should or what could we do to reduce further the incidence of immediate ICM-hypersensitivity reactions? Currently, this is a central question in clinical radiology. I think there are the following options to achieve this goal (Table 1). 
Table 1 Actions to lower the incidence of ICM-hypersensitivity reactions

Use low-ICM dose CT examinations

Lower the injection speed of ICM

Reduce the environmental contamination with ICM

Search and identify the culprit agent (sometimes non-ICM-substances induce the hypersensitivity)

Exactly document each ICM-hypersensitivity reaction

Use correct terms for the observed reactions (type A and type B reactions instead of physiological and allergy-like reaction), and omit "iodine allergy"

(i) As shown by Park and colleagues (1), lowering both the ICM-dose and the injection speed is a reliable method to decrease the incidence of immediate ICM-hypersensitivity reactions. We should broadly perform this in patients undergoing contrast-enhanced CTexaminations all over the world;

(ii) Another method to reduce the incidence of ICM-hypersensitivity reactions could be the reduction of the environmental contamination with ICM. Iodinated contrast materials are renal eliminated in chemical intact form. Therefore, via wastewater ICMs reach the environment and contaminate ground and drinking water (2). Uptake of ICM-contaminated drinking water could be a possible source for a (silent) ICMsensitization. Following this event, ICMinjection for contrast-enhanced procedures could lead in such individuals to clinical manifest ICM-hypersensitivity reactions. This means, the reduction of the ICM-dose for contrast-enhanced radiological procedures has in the long run and far-reaching consequences;

(iii) Patients reacting in a setting of contrastenhanced procedures are suspected mainly or only to have an ICM-related adverse reaction/ hypersensitivity. Usually, radiologists do not perform a detailed search for the true culprit agent. As shown previously, in nearly $75 \%$, adverse reactions are caused by contrast agents, and in $\sim 10 \%$, these reactions have other reasons (3). Consequently, it is useful to search for the individual relevant cause in patients suspected for ICM-hypersensitivity. In other words, without an exclusion of non-ICM culprit agents the mentioned incidences could be overestimated;

(iv) Exact documentations of ICM-hypersensitivity reactions enable us to perform an individual management program followed by a safe ICMapplication. Such actions also decrease the incidence of ICM-hypersensitivity (4). The more exact the documentation, the more exact the subsequent individual management. Although recommendations concerning the documentation are boring, they are very important for the patients' safety. We should document the three following parameters: date of the adverse reaction, name of the culprit ICM/agent, and the clinical symptoms of the adverse reaction (5). Exact documentation includes the use of correct terms for the observed hypersensitivity. The term "iodine allergy" for example—should be omitted, because such kind of allergy does not exist $(4,6)$. Moreover, the term is confusing, and leads to ineffective actions. In addition, there are still other terms like "allergy-like" and "physiological" reaction that are also inexact and confusing which should be replaced by exacter ones (6-9). Last but not least, the exact documentation of skin reactions could be challenge. Therefore, eligible overview articles inform radiologist about them (10).

(II) Currently, the minimal possible incidence of immediate ICM-hypersensitivity reactions is unknown. Nevertheless, the incidence is low in these days. Currently, contrast media have the lowest rate of adverse events of all drugs. This general accepted fact clearly points to another circumstance, namely to the nature of the reactions. The lower the incidence the greater the likelihood of an allergy rather than an allergy-like reaction. Unfortunately, 
even without allergy-workout immediate hypersensitivity reactions still are called 'allergylike' (6). Since this is incorrect and confusing, the term 'hypersensitivity reaction' is much more suitable to describe such reactions. Of course, in the cohort of ICM-hypersensitivity reactions there will be both, patients with true allergy and non-allergy reactions. We should be aware about this fact, and should always anticipate the worst case, namely the allergy. Moreover, a low percentage of patients with hypersensitivity reaction points to a special reactivity rather than to a simple histamine release as discussed by Park et al. (1).

(III) When comparing control and interventional period, Park et al. clearly showed a significant decrease of moderate to severe reactions $(\mathrm{P}=0.004)$, while mild reactions decreased not significantly (1). This was surprising. Because this is, exact the opposite of my anticipation. Possibly, a detailed analysis of the subgroup ( $n=6,207$ patients) that was examined in both periods [i.e., the control and the intervention period (1)], could help to understand better why patients reacted and why not.

Taken together, the recently published work by Park et al. (1) is a milestone paper. The authors clearly demonstrated in a great patient cohort that lowering both the ICM-dose as well as the injection speed rate, is accompanied by a significant drop of the incidence of immediate ICM-hypersensitivity reactions.

\section{Acknowledgments}

Funding: None.

\section{Footnote}

Conflicts of Interest: The author has completed the ICMJE uniform disclosure form (available at http://dx.doi. org/10.21037/qims.2020.03.09). The author has no conflicts of interest to declare.

Open Access Statement: This is an Open Access article distributed in accordance with the Creative Commons Attribution-NonCommercial-NoDerivs 4.0 International License (CC BY-NC-ND 4.0), which permits the noncommercial replication and distribution of the article with the strict proviso that no changes or edits are made and the original work is properly cited (including links to both the formal publication through the relevant DOI and the license). See: https://creativecommons.org/licenses/by-nc-nd/4.0/.

\section{References}

1. Park HJ, Son JH, Kim TB, Kang MK, Han K, Kim EH, Kim AY, Park SH. Relationship between Lower Dose and Injection Speed of Iodinated Contrast Material for CT and Acute Hypersensitivity Reactions: An Observational Study. Radiology 2019;293:565-72.

2. Böhm I. Iodinated X-ray contrast media in aquatic environment in general and in drinking water in particular: A possible source for the primary sensitization of patients. Chemosphere 2018;194:28-9.

3. Böhm I, Alfke H, Klose KJ. Hypersensitivity reactions and contrast medium injection: are they always related? Eur J Radiol 2011;80:368-72.

4. Böhm I, Nairz K, Morelli JN, Keller PS, Heverhagen JT. Iodinated Contrast Media and the Alleged "Iodine Allergy": An Inexact Diagnosis Leading to Inferior Radiologic Management and Adverse Drug Reactions. Rofo 2017;189:326-32.

5. Boehm I. Three Important Points on the Documentation of Contrast Hypersensitivity Reactions to Improve Contrast Medium Safety. J Am Coll Radiol 2020;17:207.

6. Böhm I, Morelli J, Nairz K, Silva Hasembank Keller P, Heverhagen JT. Myths and misconceptions concerning contrast media-induced anaphylaxis: a narrative review. Postgrad Med 2017;129:259-66.

7. Boehm I, Lombardo P. Letter to the Editor: how to document adverse reactions induced by gadolinium based contrast agents? A plea for type A and type B reactions. Eur Radiol 2020;30:1755-6.

8. Lombardo P, Boehm I. Physiological reaction following contrast medium administration: What kind of reaction is this? Eur J Intern Med 2019;62:e15.

9. Böhm I, Heverhagen JT, Klose KJ. Classification of acute and delayed contrast media-induced reactions: proposal of a threestep system. Contrast Media Mol Imaging 2012;7:537-41.

10. Böhm I, Schild HH. A practical guide to diagnose lesserknown immediate and delayed contrast media-induced adverse cutaneous reactions. Eur Radiol 2006;16:1570-9.

Cite this article as: Böhm IB. Lower dose and lower injection speed of iodinated contrast media: a new strategy to reduce the incidence rate of immediate hypersensitivity reactions. Quant Imaging Med Surg 2020;10(4):883-885. doi: 10.21037/ qims.2020.03.09 